\title{
Recuperação do centro de rotação do quadril com tântalo em artroplastias de revisão*
}

\section{Recovery of the Hip Rotation Center with Tantalum in Revision Arthroplasty}

\author{
Antônio Augusto Guimarães Barros ${ }^{1}$ (1) Victor Atsushi Kasuya Barbosa ${ }^{1} \quad$ Lincoln Paiva Costa $^{1}$ \\ Euler de Carvalho Guedes ${ }^{1}$ Carlos César Vassalo ${ }^{1}$ \\ ${ }^{1}$ Hospital Madre Teresa, Belo Horizonte, MG, Brasil \\ Rev Bras Ortop 2019;54:471-476. \\ Endereço para correspondência Antônio Augusto Guimarães Barros, \\ Alameda do Morro, 85. Nova Lima, Minas Gerais, Brasil. CEP:34006083 \\ (e-mail: antonioagbarros@gmail.com).
}

\section{Resumo \\ Palavras-chave \\ - artroplastia de quadril \\ - acetábulo \\ - tântalo}

Abstract
Objetivo O objetivo do presente estudo é avaliar a capacidade de restauração do centro de rotação anatômico do quadril com uso de copas acetabulares de tântalo associado ou não a cunhas de adição.

Métodos Análise retrospectiva dos pacientes submetidos a revisão de artroplastia do quadril com uso de tântalo entre o período de junho de 2013 e abril de 2017. Foram avaliados o ângulo de abdução do componente acetabular e as distâncias horizontal e vertical do componente ao centro de rotação anatômico do quadril. As medidas foram realizadas através de radiografias da bacia realizadas no pré-operatório e na última visita de seguimento.

Resultados Obteve-se uma amostra de 21 pacientes, 11 (52\%) homens e 10 (48\%) mulheres, com média de idade de $62 \pm 13$ anos. $O$ ângulo médio de abdução da copa acetabular reduziu de $48,76^{\circ} \pm 13,88^{\circ}$ no pré-operatório para $38,52^{\circ} \pm 10,08^{\circ}$ no pósoperatório, sendo esta diferença estatisticamente significativa $(p=0,001)$. As distâncias do centro de rotação da prótese em relação ao centro de rotação anatômico do quadril também foram menores após a cirurgia de revisão com o tântalo. A distância média horizontal de $12,74 \pm 10,59 \mathrm{~mm}$ foi reduzida para $7,11 \pm 4,84 \mathrm{~mm}$, e a distância média vertical foi reduzida de $14,79 \pm 10,05 \mathrm{~mm}$ para 4,89 $\pm 6,21 \mathrm{~mm}$, sendo essas reduções estatisticamente significativas ( $p<0,001)$.

Conclusão As revisões de artroplastia do quadril com copas de tântalo, associadas ou não a cunhas de adição, recuperaram de forma significativa o centro de rotação anatômico do quadril.

Objective The objective of the present study is to evaluate the restoration capacity of the hip anatomic rotation center with the use of acetabular tantalum cups, associated or not with addition wedges.

Trabalho realizado no Hospital Madre Teresa, Belo Horizonte, MG, Brasil.

(D) Antônio Augusto Guimarães Barros's ORCID is https://orcid.org/ 0000-0003-3701-1937.

recebido

14 de Março de 2018

aceito

21 de Agosto de 2018
DOI https://doi.org/

10.1055/s-0039-1693047. ISSN 0102-3616.
Copyright $\odot 2019$ by Sociedade Brasileira License terms de Ortopedia e Traumatologia. Published by Thieme Revnter Publicações Ltda, Rio de Janeiro, Brazil 


\author{
Keywords \\ - arthroplasty, \\ replacement, hip \\ - acetabulum \\ - tantalum
}

Methods Retrospective analysis of patients undergoing hip arthroplasty revision using tantalum between June 2013 and April 2017. The abduction angle of the acetabular component and the horizontal and vertical distances of the component to the center of anatomical rotation of the hip were evaluated. The measurements were made through baseline radiographs performed in the preoperative period and at the last follow-up visit.

Results A sample of 21 patients was obtained, 11 (52\%) men and 10 (48\%) women, with a mean age of $62 \pm 13$ years old. The mean abduction angle of the acetabular cup decreased from $48.76^{\circ} \pm 13.88^{\circ}$ in the preoperative period to $38.52^{\circ} \pm 10.08^{\circ}$ in the postoperative period, and this difference was statistically significant $(p=0.001)$. The distances from the center of rotation of the prosthesis relative to the center of anatomical rotation of the hip were also lower after revision surgery with tantalum. The mean horizontal distance of $12.74 \pm 10.59 \mathrm{~mm}$ was reduced to $7.11 \pm 4.84 \mathrm{~mm}$, and the mean vertical distance was reduced from $14.79 \pm 10.05 \mathrm{~mm}$ to $4.89 \pm 6.21 \mathrm{~mm}$, and these reductions were statistically significant $(p<0.001)$.

Conclusion Hip arthroplasty revision with tantalum cups, associated or not with addition wedges, significantly recovered the anatomical rotation center of the hip.

\section{Introdução}

Há uma tendência no aumento das revisões de artroplastia total do quadril (RATQs) nos próximos anos. Nos EUA, é esperado que o número de RATQs dobre no período entre 2005 e 2026. ${ }^{1}$ As causas mais comuns de revisão são instabilidade (17,3\%), soltura asséptica (16,8\%), complicações mecânicas não especificadas $(13,4 \%)$ e infecção $(12,8 \%){ }^{2}$ Na maioria dos casos, o componente acetabular é substituído, sendo todos os componentes trocados em $40,3 \%$ dos casos e o acetábulo isoladamente em $14,5 \%$ das reoperações. ${ }^{2}$ A revisão desse componente com restauração do centro anatômico de rotação do quadril, correção dos defeitos ósseos acetabulares e obtenção de uma estabilidade primária da copa é um desafio para o cirurgião ortopédico. ${ }^{3}$ As técnicas mais comumente descritas são o uso de copas convencionais sem cimento de diâmetro maior ou extragrandes, ${ }^{4}$ copas cimentadas com ou sem enxerto impactado, ${ }^{5}$ anel de reforço acetabular, ${ }^{6}$ construção do tipo cup-cage ${ }^{7}$ e o uso de copas com metais de alta porosidade (por exemplo, tântalo) associado ou não a cunhas de adição. ${ }^{8}$ Apesar da quantidade de opções disponíveis, essas técnicas ainda apresentam resultados variáveis de acordo com o grau de lesão óssea, podendo apresentar falha em até 88,5\% dos casos após seguimento médio de 44,6 meses. ${ }^{9}$

Mesmo nos casos de revisões complexas com grande perda óssea, os implantes de revisão revestidos com metal de alta porosidade vêm apresentando bons resultados nos seguimentos de curto e médio prazo. ${ }^{10,11}$ Este material apresenta vantagens teóricas em relação aos demais materiais, tais como: alto coeficiente de atrito, módulo de elasticidade mais próximo do osso e grande volume de porosidade, permitindo uma capacidade maior de osteointegração e uma melhor fixação secundária da copa. ${ }^{12,13}$ Porém, são materiais relativamente novos e caros, onerando consideravelmente o sistema de saúde. O objetivo do presente estudo é avaliar a capacidade de restauração do centro de rotação anatômico do quadril com uso de copas acetabulares revestidas com metal de alta porosidade, associado ou não a cunhas de adição. Nossa hipótese é a de que as RATQs realizadas com metal de alta porosidade foram eficazes em melhorar o posicionamento do centro de rotação do quadril ao se comparar aos valores recomendados na literatura.

\section{Material e Método}

Utilizando o banco de dados de artroplastia do hospital da nossa instituição, foram identificados todos os pacientes que foram submetidos a RATQ no período de junho de 2013 e abril de 2017. Após a aprovação pelo comitê de ética institucional, foram convidados a participar do estudo os pacientes submetidos a reconstrução cirúrgica acetabular utilizando componentes com revestimento de tântalo associado ou não a cunhas de adição (Trabecular Metal, Zimmer, Warsaw, Indiana, USA), para realização de uma análise retrospectiva. Foram incluídos no estudo todos os pacientes que realizaram RATQ na unidade hospitalar no período de junho de 2013 a abril de 2017, com uso do tântalo, independente do motivo da revisão, do grau de perda óssea ou da presença de qualquer comorbidade. Foram excluídos apenas pacientes que foram a óbito ou não realizaram segmento adequado.

Os defeitos prévios à cirurgia foram descritos conforme a classificação de Paprosky et al, ${ }^{14}$ e os parâmetros radiográficos foram medidos através de radiografias da bacia (AP e oblíquas) realizadas durante o período pré-operatório e na última visita de seguimento. Foram medidos o ângulo de abdução do componente acetabular, em relação à gota de lágrima ou à tuberosidade isquiática, assim como as distâncias horizontal e vertical do componente ao centro de rotação anatômico do quadril. A partir da altura pélvica, foi traçado um triangulo isósceles lateralmente a $5 \mathrm{~mm}$ do cruzamento entre a linha de 
Shenton e a linha de Koehler, em que os lados do triangulo medem $20 \%$ da altura pélvica. 0 centro de rotação é definido como a metade do comprimento da hipotenusa. ${ }^{15}$

\section{Análise Estatística}

As variáveis categóricas foram apresentadas em valor absoluto e percentual, e as contínuas por média e desvio padrão (DP). As variáveis contínuas foram submetidas à avaliação da normalidade por meio do teste de Shapiro-Wilk. A comparação entre os períodos pré- e pós-operatórios foi feita com o teste $t$ de Student pareado para as variáveis de distribuição paramétrica. No caso de amostras independentes, a comparação de dois grupos foi realizada através do teste $t$ de Student para amostras independentes, após confirmada a distribuição normal, e avaliação das variâncias (ANOVA) pelo teste de Levene. $O$ coeficiente de correlação entre valores pré- e pós-operatório das variáveis contínuas foi feito com o teste de Spearman. A força da correlação foi classificada de acordo com o valor de $\mathrm{r}$, sendo forte se $>0,70$, moderada entre 0,30 e 0,70 e fraca se $<0,30$. Usamos para análise dos dados o programa SPSS Satistics for Windows, Versão 20.0 (IBM Corp., Armonk, NY, EUA) e nível de significância de 5\%.

\section{Resultados}

Entre o período de junho de 2013 e abril de 2017 foram realizadas 95 RATQs. Deste total, 21(22\%) pacientes foram submetidos a revisão acetabular com copa revestida de tântalo, associado ou não a cunhas de adição (Trabecular Metal, Zimmer, Warsaw, Indiana, USA). Foi observada uma tendência no aumento do uso desse material na nossa amostra (-Fig. 1). As características da amostra estão apresentadas na - Tabela 1.

0 ângulo médio de abdução da copa acetabular reduziu de $48,76^{\circ} \pm 13,88^{\circ}$ no pré-operatório para $38,52^{\circ} \pm 10,08^{\circ}$ no pós-operatório, sendo esta diferença estatisticamente significativa $(p=0,001)$. As distâncias do centro de rotação da prótese em relação ao centro de rotação anatômico do quadril também foram menores após a cirurgia de revisão com o uso do componente acetabular de tântalo. A distância média horizontal de $12,74 \pm 10,59 \mathrm{~mm}$ foi reduzida para $7,11 \pm 4,84 \mathrm{~mm}$, e a distância média vertical de $14,79 \pm 10,05 \mathrm{~mm}$ foi reduzida para 4,89 $\pm 6,21 \mathrm{~mm}$, sendo estas reduções estatisticamente significativas $(p<0,001)(-$ Tabela 2$)$.

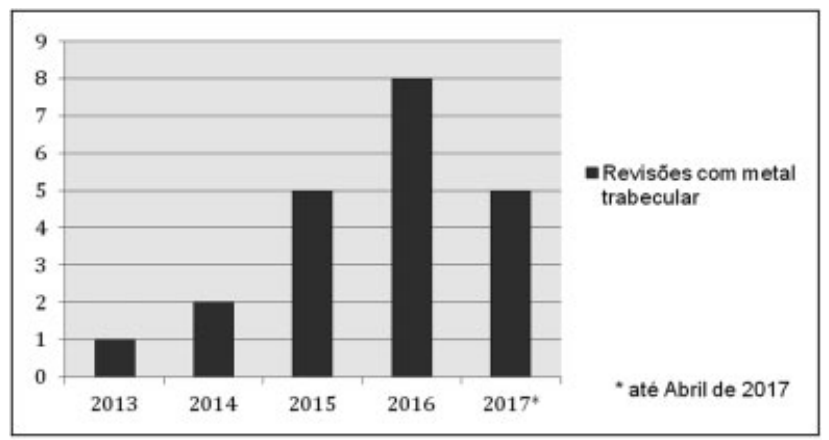

Fig. 1 Uso do metal trabecular nos últimos 5 anos.
Tabela 1 Características da amostra

\begin{tabular}{|c|c|}
\hline Pacientes, $n$ & 21 \\
\hline \multicolumn{2}{|l|}{ Gênero, $n$ (\%) } \\
\hline Masculino & $11(52,4 \%)$ \\
\hline Feminino & $10(47,6 \%)$ \\
\hline Idade média, anos (desvio padrão) & $62( \pm 13,2)$ \\
\hline \multicolumn{2}{|l|}{ Indicação da revisão, n (\%) } \\
\hline Soltura asséptica & $15(71,4 \%)$ \\
\hline Instabilidade & $1(4,8 \%)$ \\
\hline Infecção & $3(14,3 \%)$ \\
\hline Dor & $2(9,5 \%)$ \\
\hline \multicolumn{2}{|l|}{ Número de revisões prévias, $n(\%)$} \\
\hline 0 & $11(52,4 \%)$ \\
\hline 1 & $8(38,1 \%)$ \\
\hline 2 & $2(9,5 \%)$ \\
\hline \multicolumn{2}{|l|}{ Classificação Paprosky, n (\%) } \\
\hline Tipo 1 & $3(14,3 \%)$ \\
\hline Tipo 2a & $3(14,3 \%)$ \\
\hline Tipo 2B & $6(28,6 \%)$ \\
\hline Tipo 2C & $0(0 \%)$ \\
\hline Tipo 3a & $2(9,5 \%)$ \\
\hline Tipo 3B & $2(9,5 \%)$ \\
\hline Descontinuidade pélvica & $5(23,8 \%)$ \\
\hline
\end{tabular}

Houve forte correlação direta entre os valores pré- e pósoperatórios da distância horizontal $(\mathrm{r}=0,928 ; p<0,001)$ e da distância vertical $(0,792 ; p<0,001)$. Portanto, a presença antes da revisão de uma maior distância até o centro de rotação anatômico do quadril foi associada também a uma maior distância após a cirurgia, indicando que desvios maiores do centro de rotação apresentam correções menores no pósoperatório (-Tabela 3).

Ao se comparar os resultados radiográficos pós-operatórios entre os grupos sem revisão prévia (11 pacientes) e com revisão prévia (10 pacientes), não foi observada diferença estatisticamente significativa entre os ângulos de abdução da copa ou entre as distâncias ao centro de rotação anatômico do quadril (-Tabela 4).

\section{Discussão}

O presente estudo mostrou que o uso de copas acetabulares revestidas de tântalo, associadas ou não a cunhas de adição, foram eficazes em recuperar o centro de rotação anatômico do quadril em cirurgias de RATQ ( - Figs. 2a, 2b, 3a e 3b). Com isso, a hipótese do presente estudo foi corroborada.

O posicionamento acetabular correto é fundamental para o sucesso da artroplastia total do quadril (ATQ) podendo influir na carga submetida à articulação e no desgaste do implante. Em relação ao centro de rotação anatômico do quadril, cada milímetro de lateralização e de proximalização da copa está 
474 Recuperação do centro de rotação do quadril Barros et al.

Tabela 2 Resultados radiológicos da mecânica do quadril

\begin{tabular}{|l|l|l|l|}
\hline & Pré-operatório & Pós-operatório & valor-p \\
\hline Ângulo de abdução da copa acetabular*, média \pm desvio padrão & $48,76 \pm 13,88$ & $38,52 \pm 10,08$ & $\mathrm{p}=0,001^{\mathrm{a}}$ \\
\hline Distância horizontal $^{* *}$, média \pm desvio padrão & $12,74 \pm 10,59$ & $7,11 \pm 4,84$ & $\mathrm{p}<0,001^{\mathrm{a}}$ \\
\hline Distância vertical $^{* *}$, média \pm desvio padrão & $14,79 \pm 10,05$ & $4,89 \pm 6,21$ & $\mathrm{P}<0,001^{\mathrm{a}}$ \\
\hline
\end{tabular}

*Valor em graus.

**Valor em mm.

${ }^{\mathrm{a}}$ Teste $\mathrm{t}$ de Student pareado.

Tabela 3 Correlação entre os valores radiológicos pré- e pós-operatórios ${ }^{\text {a }}$

\begin{tabular}{|l|l|l|}
\hline & Valor de $\mathbf{r}$ & valor-p \\
\hline Ângulo de abdução da copa acetabular & 0,525 & $\mathrm{p}=0,015$ \\
\hline Distância horizontal & 0,928 & $\mathrm{p}<0,001$ \\
\hline Distância vertical & 0,792 & $\mathrm{p}<0,001$ \\
\hline
\end{tabular}

${ }^{a}$ Coeficiente de correlação de Spearman.

Tabela 4 Comparação dos resultados radiológicos pós-operatório entre os grupos sem e com revisões prévias

\begin{tabular}{|c|c|c|c|}
\hline & $\begin{array}{l}\text { Ausência de } \\
\text { revisão prévia }\end{array}$ & $\begin{array}{l}\text { Presença de } \\
\text { revisão prévia }\end{array}$ & valor-p \\
\hline Ângulo de abdução da copa acetabular*, média \pm desvio padrão & $36,09 \pm 11,29$ & $41,20 \pm 8,29$ & $p=0,256^{a}$ \\
\hline Distância horizontal**, média \pm desvio padrão & $6,36 \pm 3,66$ & $7,94 \pm 5,98$ & $\mathrm{p}=0,470^{\mathrm{a}}$ \\
\hline Distância vertical ${ }^{* *}$, média \pm desvio padrão & $3,65 \pm 6,37$ & $6,24 \pm 6,06$ & $p=0,354^{a}$ \\
\hline
\end{tabular}

*Valor em graus.

**Valor em mm.

${ }^{\mathrm{a}}$ Teste $\mathrm{t}$ de Student para amostras independents.

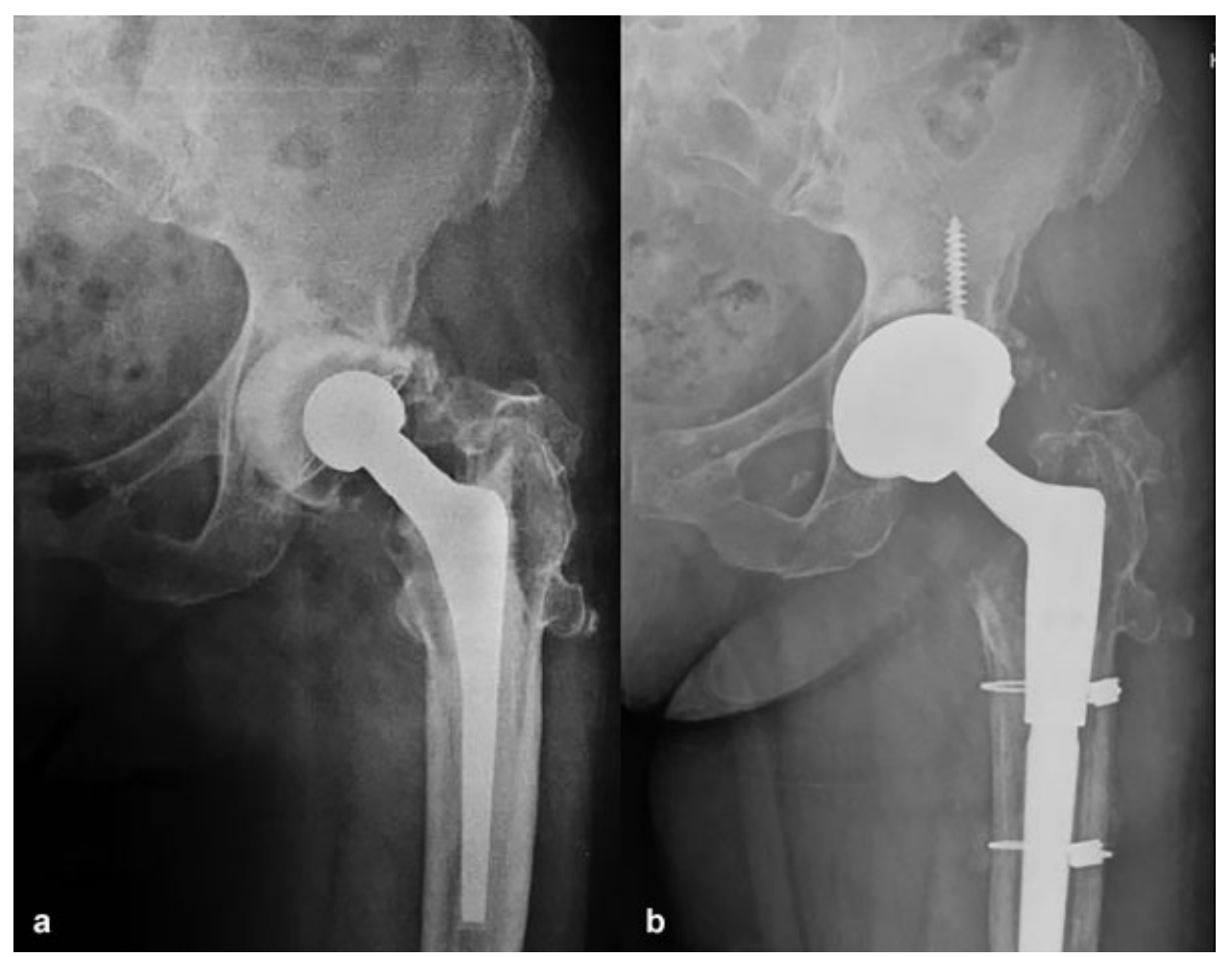

Fig. 2 Recuperação do centro de rotação do quadril com copa de tântalo sem adição de cunha. a) Radiografia pré-operatória; b) Radiografia pósoperatória. 


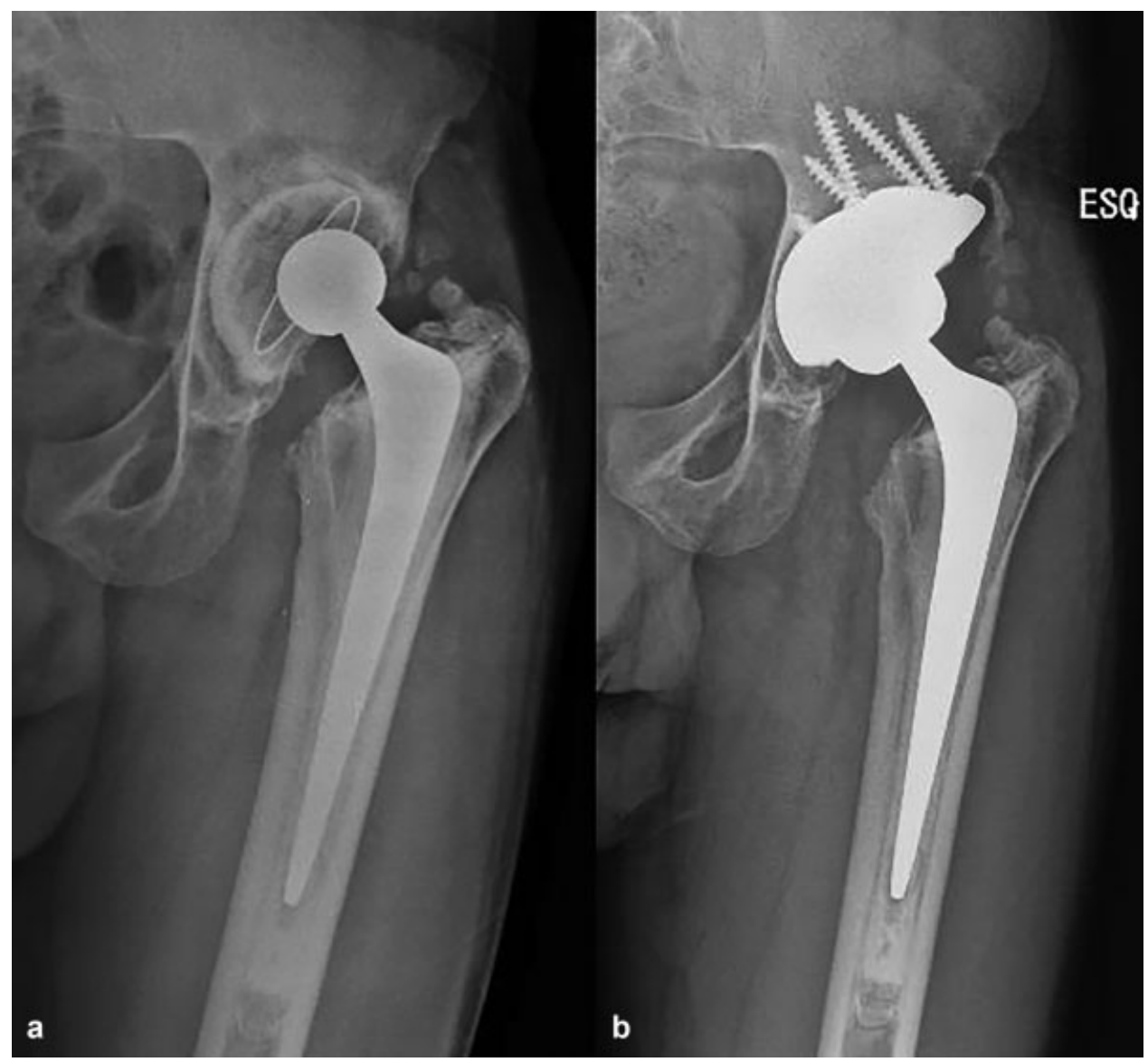

Fig. 3 Recuperação do centro de rotação do quadril com copa de tântalo e com cunha de adição. a) Radiografia pré-operatória; b) Radiografia pós-operatória.

associado a um aumento de $0,7 \%$ e de $0,1 \%$ na carga articular, respectivamente. ${ }^{16} \mathrm{Na}$ literatura há certo consenso de que a copa acetabular deve estar até no máximo $5 \mathrm{~mm}$ distante do centro de rotação anatômico do quadril. ${ }^{17-19} \mathrm{O}$ posicionamento da copa com um ângulo $>45^{\circ}$ esteve associado a um aumento de $40 \%$ no desgaste linear médio do polietileno. ${ }^{20} \mathrm{O}$ posicionamento mais horizontal da copa acetabular pode reduzir a osteólise pélvica. Kennedy et al, ${ }^{21}$ ao avaliarem 75 pacientes submetidos a ATQ divididos em 2 grupos com abdução média de $61,9^{\circ}$ e de $49,7^{\circ}$, observaram taxas de osteólise pélvica de $24 \%$ e de $13 \%$ nos grupos de maior e menor inclinação acetabular, respectivamente, após 4 anos de seguimento.

A melhora do posicionamento do centro de rotação do quadril pode ser favorável em relação aos resultados funcionais, ${ }^{22}$ já que o centro de rotação é muito importante para a função muscular. Asayama et al, ${ }^{23}$ ao estudarem 30 pacientes com ATQ encontraram correlação negativa entre a força da musculatura abdutora e a proximalização do centro de rotação do quadril. Outro estudo, através de análise biomecânica de um modelo de membro inferior, mostrou que o desvio superior de $2 \mathrm{~cm}$ esteve relacionado a uma diminuição de $44 \%$ na força abdutora. ${ }^{24} \mathrm{~A}$ fraqueza da musculatura abdutora do quadril foi uma das causas mais importantes de luxação da prótese em um estudo envolvendo 1.318 pacientes. ${ }^{25}$
Abolghasemian et $\mathrm{al}^{26}$ corroboram os resultados de melhora do posicionamento do centro de rotação do componente acetabular após revisão com metal de alta porosidade. Houve melhora da posição do centro de rotação em ambos os eixos após a revisão, de modo que a distância média do centro anatômico antes da revisão era de $28,8 \mathrm{~mm}$ $(-3 \mathrm{~mm}$ a $79 \mathrm{~mm})$ no eixo vertical, e de $13,3 \mathrm{~mm}(-21 \mathrm{~mm}$ a $35 \mathrm{~mm}$ ) no eixo horizontal. 0 pós-operatório apresentou distância média do centro de rotação de $9,9 \mathrm{~mm}(-18 \mathrm{~mm}$ a $37 \mathrm{~mm})$ superior e de $5,1 \mathrm{~mm}(-25 \mathrm{~mm}$ a $30 \mathrm{~mm})$ lateral ao centro anatômico. Jenkins et $\mathrm{al}^{10}$ também relataram recuperação do centro de rotação do quadril. A distância horizontal média ao centro de rotação do quadril reduziu de $9 \mathrm{~mm}$ no pré-operatório (intervalo de $1 \mathrm{~mm}$ a $45 \mathrm{~mm}$ ) para $8 \mathrm{~mm}$ no pós-operatório (intervalo de $0 \mathrm{~mm}$ a $27 \mathrm{~mm}$ ) $(p=0,0143$ ), e esta distância foi de $9 \mathrm{~mm}$ (intervalo de $0 \mathrm{~mm}$ a $26 \mathrm{~mm}$ ) no último acompanhamento. A distância vertical média ao centro de rotação do quadril diminuiu de $21 \mathrm{~mm}$ no pré-operatório (intervalo de $0 \mathrm{~mm}$ a $80 \mathrm{~mm}$ ) para $6 \mathrm{~mm}$ no pós-operatório (intervalo de $0 \mathrm{~mm}$ a $28 \mathrm{~mm}$ ) $(p=0,0001)$ e para $4,5 \mathrm{~mm}$ (intervalo de $0 \mathrm{~mm}$ a $57 \mathrm{~mm}$ ) no último seguimento. 0 ângulo médio de abdução da copa acetabular foi reduzido de $61^{\circ}$ no pré-operatório (intervalo, $-18^{\circ}$ a $\left.180^{\circ}\right)$ para $45^{\circ}$ no pós-operatório (intervalo de $34^{\circ}$ a $\left.60^{\circ}\right)(p=0,0001)$ e para $45^{\circ}$ (intervalo de $34^{\circ}$ a $82^{\circ}$ ) no último acompanhamento. 
O presente estudo apresenta algumas limitações. Trata-se de um estudo retrospectivo e com amostra relativamente pequena, de 21 pacientes. A restauração do centro de rotação foi avaliada apenas no plano coronal, não levando em consideração desvios no sentido anteroposterior, e não foi avaliada a versão acetabular.

\section{Conclusão}

Copas acetabulares revestidas de tântalo, associadas ou não a cunhas de adição, foram eficazes em melhorar o posicionamento do centro de rotação anatômico do quadril em cirurgias de revisão de maneira significativa.

\section{Conflitos de Interesses}

Os autores declaram não haver conflitos de interesses.

\section{Referências}

1 Kurtz S, Ong K, Lau E, Mowat F, Halpern M. Projections of primary and revision hip and knee arthroplasty in the United States from 2005 to 2030. J Bone Joint Surg Am 2007;89(04):780-785

2 Gwam CU, Mistry JB, Mohamed NS, Thomas M, Bigart KC, Mont MA, et al. Current Epidemiology of Revision Total Hip Arthroplasty in the United States: National Inpatient Sample 2009 to 2013. J Arthroplasty 2017;32(07):2088-2092

3 Paprosky WG, Magnus RE. Principles of bone grafting in revision total hip arthroplasty. Acetabular technique. Clin Orthop Relat Res 1994;(298):147-155

4 Lachiewicz PF, Soileau ES. Fixation, survival, and dislocation of jumbo acetabular components in revision hip arthroplasty. J Bone Joint Surg Am 2013;95(06):543-548

5 Schreurs BW, Keurentjes JC, Gardeniers JW, Verdonschot N, Slooff TJ, Veth RP. Acetabular revision with impacted morsellised cancellous bone grafting and a cemented acetabular component: a 20- to 25-year follow-up. J Bone Joint Surg Br 2009;91(09):1148-1153

6 Mäkinen TJ, Kuzyk P, Safir OA, Backstein D, Gross AE. Role of cages in revision arthroplasty of the acetabulum. J Bone Joint Surg Am 2016;98(03):233-242

7 Mäkinen TJ, Fichman SG, Watts E, Kuzyk PR, Safir OA, Gross AE. The role of cages in the management of severe acetabular bone defects at revision arthroplasty. Bone Joint J 2016;98-B(1, Suppl A):73-77

8 Laaksonen I, Lorimer M, Gromov K, Rolfson O, Mäkelä KT, Graves $\mathrm{SE}$, et al. Does the risk of rerevision vary between porous tantalum cups and other cementless designs after revision hip arthroplasty? Clin Orthop Relat Res 2017;475(12):3015-3022

9 Kosashvili Y, Backstein D, Safir O, Lakstein D, Gross AE. Acetabular revision using an anti-protrusion (ilio-ischial) cage and trabecular metal acetabular component for severe acetabular bone loss associated with pelvic discontinuity. J Bone Joint Surg Br 2009; 91(07):870-876

10 Jenkins DR, Odland AN, Sierra RJ, Hanssen AD, Lewallen DG. Minimum five-year outcomes with porous tantalum acetabular cup and augment construct in complex revision total hip arthroplasty. J Bone Joint Surg Am 2017;99(10):e49

11 Konan S, Duncan CP, Masri BA, Garbuz DS. Porous tantalum uncemented acetabular components in revision total hip arthroplasty: a minimum ten-year clinical, radiological and quality of life outcome study. Bone Joint J 2016;98-B(06):767-771

12 Bobyn JD, Stackpool GJ, Hacking SA, Tanzer M, Krygier JJ. Characteristics of bone ingrowth and interface mechanics of a new porous tantalum biomaterial. J Bone Joint Surg Br 1999;81(05):907-914

13 Bobyn JD, Poggie RA, Krygier JJ, Lewallen DG, Hanssen AD, Lewis RJ, et al. Clinical validation of a structural porous tantalum biomaterial for adult reconstruction. J Bone Joint Surg Am 2004;86-A(Suppl 2):123-129

14 Paprosky WG, Perona PG, Lawrence JM. Acetabular defect classification and surgical reconstruction in revision arthroplasty. A 6-year follow-up evaluation. J Arthroplasty 1994;9(01):33-44

15 Pagnano W, Hanssen AD, Lewallen DG, Shaughnessy WJ. The effect of superior placement of the acetabular component on the rate of loosening after total hip arthroplasty. J Bone Joint Surg Am 1996;78(07):1004-1014

16 Bicanic G, Delimar D, Delimar M, Pecina M. Influence of the acetabular cup position on hip load during arthroplasty in hip dysplasia. Int Orthop 2009;33(02):397-402

17 Liebs TR, Nasser L, Herzberg W, Rüther W, Hassenpflug J. The influence of femoral offset on health-related quality of life after total hip replacement. Bone Joint J 2014;96-B(01):36-42

18 Cassidy KA, Noticewala MS, Macaulay W, Lee JH, Geller JA. Effect of femoral offset on pain and function after total hip arthroplasty. J Arthroplasty 2012;27(10):1863-1869

19 Jolles BM, Zangger P, Leyvraz PF. Factors predisposing to dislocation after primary total hip arthroplasty: a multivariate analysis. J Arthroplasty 2002;17(03):282-288

20 Patil S, Bergula A, Chen PC, Colwell CW Jr, D'Lima DD. Polyethylene wear and acetabular component orientation. J Bone Joint Surg Am 2003;85-A(04, Suppl 4):56-63

21 Kennedy JG, Rogers WB, Soffe KE, Sullivan RJ, Griffen DG, Sheehan LJ. Effect of acetabular component orientation on recurrent dislocation, pelvic osteolysis, polyethylene wear, and component migration. J Arthroplasty 1998;13(05):530-534

$22 \mathrm{Kim} \mathrm{DH}, \mathrm{Cho}$ SH, Jeong ST, Park HB, Hwang SC, Park JS. Restoration of the center of rotation in revision total hip arthroplasty. J Arthroplasty 2010;25(07):1041-1046

23 Asayama I, Chamnongkich S, Simpson KJ, Kinsey TL, Mahoney OM. Reconstructed hip joint position and abductor muscle strength after total hip arthroplasty. J Arthroplasty 2005;20(04):414-420

24 Delp SL, Maloney W. Effects of hip center location on the moment-generating capacity of the muscles. J Biomech 1993; 26(4-5):485-499

25 García-Rey E, García-Cimbrelo E. Abductor biomechanics clinically impact the total hip arthroplasty dislocation rate: a prospective long-term study. J Arthroplasty 2016;31(02):484-490

26 Abolghasemian M, Tangsataporn S, Sternheim A, Backstein D, Safir O, Gross AE. Combined trabecular metal acetabular shell and augment for acetabular revision with substantial bone loss: a mid-term review. Bone Joint J 2013;95-B(02):166-172 